\title{
The Evaluations of Bleach as Decontaminant Solution to Promote The Positivity Rate of Mycobacterium Tuberculosis Culture for Sputum Specimen
}

\author{
Erike A. Suwarsono \\ Microbiology Laboratory, Faculty of Medicine and Health \\ Sciences \\ Syarif Hidayatullah State Islamic University \\ Jakarta, Indonesia \\ erike.suwarsono@uinjkt.ac.id
}

\author{
Anis Karuniawati, Agus Sjahrurachman, Erlina \\ Burhan* \\ Microbiology Department, Faculty of Medicine \\ University of Indonesia \\ Jakarta, Indonesia \\ *Pulmonology Department, Faculty of Medicine \\ University of Indonesia \\ Jakarta, Indonesia
}

\begin{abstract}
Background: Tuberculosis (TB) is still worldwide health problems. Sputum is contaminated by normal flora, that make its should be decontaminated prior to culture. Bleach known as a potent disinfectant, easy to access because it is commercially available, could be the alternative solution as decontaminant in TB culture.

Aims Assessing the capability of bleach to promote the positivity rate in TB culture compared by others decontaminant solution. Method We use 35 sputum samples with volume 3-5 ml. The research was conducted at TB laboratory of Faculty of Medicine University of Indonesia, by using 35 samples sputum with positive AFB, 3-5 ml. Each sample was divided into 4 groups that were decontaminated by different methods. The methods are $4 \% \mathrm{NaOH}, 2 \% \mathrm{NALC}-\mathrm{NaOH}, 5 \%$ oxalic acid and $1 \%$ bleach. $1 \%$ bleach was prepared from commercially bleach. The positive culture was validated using MPT 64 . The culture positivity rate was compared based on its AFB score.

Result Bleach had the same $100 \%$ positivity rate as the $4 \%$ $\mathrm{NaOH}$ and NALC for $(+2)$ and $(+3)$ AFB. However, its became smaller rate for the (+1) AFB group, which was $88 \%$ for bleach and $96 \%$ for NALC and $100 \%$ for $\mathrm{NaOH}$. Oxalic acid had the smallest positivity rate in all AFB score compared to the others. Conclusion The positivity rate of the bleach as same as $4 \% \mathrm{NaOH}$ and NALC for AFB score higher than (+2)
\end{abstract}

Keywords : bleach; decontaminant; contamination; TB culture

\section{INTRODUCTION}

Tuberculosis (TB) is still one of major infectious illness in the world. In 2015. WHO reported that total incidence of TB are around 10.4 million people worldwide. ${ }^{1}$ The gold standard to diagnose TB is finding Mycobacterium tuberculosis in culture. ${ }^{2,3}$ Sputum as the most common specimen for TB culture should be decontaminated prior to culture. The decontaminant solutions that ordinary used in TB laboratory are Sodium hydroxide $(\mathrm{NaOH}), N$-acetyl-L-cysteine and
Sodium Hydroxide (NALC-NaOH) and oxalic acid. ${ }^{4}$ However, in developing countries, such as Indonesia, not all TB laboratories have capability to use those decontaminant solutions, not only because the financial burden but also the lack of chemical solution availability. Bleach as potent decontaminant and easy to get as commercially available could be an alternative decontaminant in TB culture. The aim of this study is to assess the capability of bleach as decontaminant to promote the positivity rate in $\mathrm{TB}$ culture

\section{METHOD}

\section{A. Sampel and ethical clearance}

This research was using 35 sputum samples from TB suspected patient, and had positive Acid Fast Bacili (AFB) score $(+1$ to +3$)$. All patients were admitted in Persahabatan Hospital Jakarta, Indonesia and clinically suspected as new TB case. Sampling technique was using consecutive method. The ethical clearance had approved from Ethical board of University of Indonesia-Cipto Mangunkusomo Hospital, Jakarta.

\section{B. Laboratory technique and processesing}

Each sample that is amount 3-5 ml, was divided into four groups that decontaminated by different four methods. The groups were $4 \% \mathrm{NaOH}$ (sodium hydroxide), $2 \%$ NALC-NaOH ( $N$-acetyl-L-cysteine and Sodium hydroxide), 5\% oxalic acid and $1 \%$ bleach. The first three groups were prepared from laboratory grade solutions and $1 \%$ bleach from commercially solution (Bayclin ${ }^{\circledR}$ batch number 8998899013046). The well known decontamination of three groups had did as stated in the Manual of Clinical Microbiology and previous publications. ${ }^{2,4}$ The AFB stain was stained using Ziehl-Neelsen method. The TB culture was conducted at TB laboratory of Microbiology Laboratory of Faculty of Medicine, University of Indonesia. 
The decontamination techniques of $1 \%$ bleach had optimized before in the same laboratory. Bleach was added within same value of sputum, then homogenized using vortex and incubated for 10 minutes. After 10 minutes the solution was neutralized and centrifuged at $3000 \mathrm{xg}, 4^{\circ} \mathrm{C}$ for 15 minutes, followed by inoculation in one paired Löwenstein-Jensen (LJ) and incubated in $35-37^{\circ} \mathrm{C}$ for $6-8$ weeks. MPT 64 was using to validate that the grown colonies was Mycobacterium tuberculosis complex. Each positive LJ was counted for positive TB culture and had compared to each other based on its own AFB score. AFB score was based on International Union Against Tuberculosis and Lung Diseases (IUATLD) criteria. As it was paired LJ from the same sputum, one or both positive LJ's tubes counted as single positive result.

\section{RESULT}

From total sample there were $46 \%(\mathrm{n}=16)$ AFB score was $(+1), 37 \%(n=13)$ for AFB $(+2)$ and $17 \%(n=6)$ for AFB score $(+3)$. Although all samples were positive AFB but the recovery rate of Mycobacterium tuberculosis of each group were not $100 \%$. Only decontamination process that was using $4 \% \mathrm{NaOH}$ can keep Mycobacterium tuberculosis still survives in all samples.

In the table 1 , we can see that the positivity rate of $\mathrm{TB}$ culture is different within each group. The NALC-NaOH took the second place for positivity rate that was mean there was $2 \%$ sample didn't recover for the Mycobacterium tuberculosis growth. In bleach group there was 3\% of samples didn't recover for the Mycobacterium tuberculosis growth. The 5\% oxalic acid have the lowest positivity rate, and make this group becomes the worst in recovery rate of Mycobacterium tuberculosis.

However based on AFB score grouping, we can see in figure 1, that the positivity rate is different based on its AFB score although the sample had come from the same sputum. The lower AFB score is linier to the positivity rate. Exception for $4 \% \mathrm{NaOH}$, all samples are $100 \%$, but for bleach, $100 \%$ positivity rate only happens in the $(+2) \mathrm{AFB}$ and (+3) AFB, and only $88 \%$ positivity rate when the AFB score is $(+1)$.

If the bleach group's result compared to the NALC-NaOH group it looks that the positivity rate between both is differ 6 points ( $88 \%$ to $94 \%)$. However the difference between bleach and oxalic acid is quite high (more than 15 points in each AFB score), which means that bleach is better that oxalic acid in recovering Mycobacterium tuberculosis.

Table 1 . The positivity rate of TB culture within each group of different decontaminant

\begin{tabular}{|c|c|c|}
\hline NO & Decontaminant group & $\begin{array}{c}\text { positivity rate }^{\mathrm{a}} \\
\text { n }(\%)\end{array}$ \\
\hline 1 & $4 \% \mathrm{NaOH}$ & $35(100)$ \\
\hline 2 & $2 \%$ NALC-NaOH & $34(97)$ \\
\hline 3 & $5 \%$ oxalic acid & $28(80)$ \\
\hline 4 & $1 \%$ bleach & $33(94)$ \\
\hline
\end{tabular}

a. positivity rate is not counted for individually positive AFB score

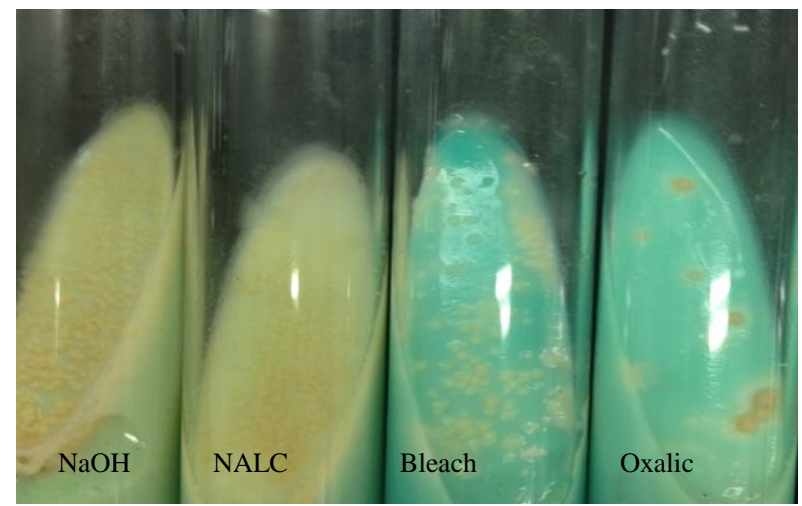

Figure 1. The TB colonies in each decontaminant method from the same sputum with AFB (+1)

\section{The positivity rate based on AFB score}

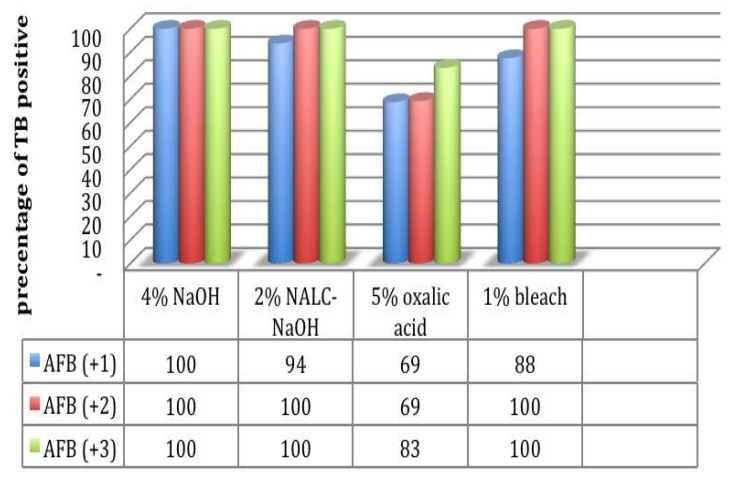

Figure 2. The positivity rate of TB culture within each group of different decontaminant based on sputum's AFB score

Bleach

\section{DISCUSSION}

Based on the result, the capability of each decontaminant solution on recovering Mycobacterium tuberculosis bacilli is differ one to another. We can see from table 1 , that $4 \% \mathrm{NaOH}$ have the best rate in recovering Mycobacterium tuberculosis as its positivity rate is $100 \%$. Many studies had already found that $\mathrm{NaOH}$ have a good positivity rate higher than $80-90 \% .^{4-6}$ Adding decontaminant prior to TB culture from sputum can minimize contamination of oropharyngeal's normal flora. ${ }^{7,8}$ Those contaminants can mask Mycobacterium tuberculosis growth.

Thus, NALC-NaOH also have positivity rate higher than $90 \%$, although the positivity rate for $\mathrm{AFB}(+2$ and +3$)$ can reach $100 \%$, but for AFB $(+1)$, it is only reach $94 \%$. Same case with the $1 \%$ bleach result, the positivity rate become lower for AFB (+1). In this case of study, NALC-NaOH have lower rate for AFB $(+1)$, because of the great number of contamination (result not shown in this paper) in the TB culture. Chaterjje, 2013 had shown that the contamination rate for NALC method was also higher than $4 \% \mathrm{NaOH}$ only. ${ }^{4}$ Another study also found that $2 \%$ NALC-NaOH more ease to be contaminated rather than the $4 \% \mathrm{NaOH}$ method. ${ }^{6,9} \mathrm{NALC}$ is a good agent as mucolytic, but not as decontaminant, this factor could lead the capability of NALC-NaOH in recovery rate is weaker than 
bleach or $\mathrm{NaOH}$ and mask the growth of Mycobacterium tuberculosis. ${ }^{2,6,10}$ Based on the number of contamination rate, $5 \%$ oxalic acid that had low number of positivity rate was also had a great number in contamination. The contamination that happened in oxalic group, could be because oxalic acid is a poor decontaminant for fungi and Gram positive bacteria as both are abundant flora in oropharyngeal. However, it's a good decontaminant for Gram negative bacteria especially Pseudomonas spp. .,4 $^{2,4}$

Different from NALC-NaOH and oxalic groups, the low number of $1 \%$ bleach in recovering Mycobacterium tuberculosis from AFB (+1) could be caused by a strong decontaminant's effect of bleach and a low number of bacilli that found per milliliter sputum of AFB $(+1)$. As we know that, there is a lower bumber of bacilli in $(+1)$ AFB rather than $(+2)$ or (+3) AFB., ${ }^{2,11}$ This condition can lead the toxic effect of bleach becomes greater to the bacilli, and can kill more numerous bacilli than in the sputum with $(+2)$ or $(+3)$ AFB. The mode of how bleach can effectively kill contaminant microorganism in TB culture by initiating oxidative stress to bacterial proteins. Those oxidative stress reactions can stimulate aggregation of its protein and resulted in death. ${ }^{12}$

From figure 1, we can assume that the number of TB colonies that had grown from $(+1)$ AFB from bleach group is smaller than $4 \% \mathrm{NaOH}$ and $2 \%$ NALC-NaOH. The factors that cause this condition maybe because the bleach concentration is too strong and time contact to the TB bacilli is too long. The concentration of chemical substance of the decontaminant and time contact to bacilli is a crucial factor for Mycobacterium tuberculosis' recovery. ${ }^{2,10,13}$ The higher concentration the more toxic to the bacilli's cells, whatever the chemical substance used to decontaminate, it is still have probability to kill a number of TB bacilli. ${ }^{2,14,15}$ As a number of bacilliin $(+1)$ AFB is lower than $(+2)$ and $(+3)$ and the bleach concentration and time contact doesn't change, it can make the recovery capability in $(+1)$ AFB is lower too.

However from figure 2 , we can conclude that, for $(+2)$ and (+3) AFB, $1 \%$ bleach can recover all of Mycobacterium tuberculosis from the sputum, and made positivity rate $100 \%$. This result seems that the concentration of bleach and time contact for incubation is properly conducted. The concentration of bleach at $1 \%$ and 10 minutes incubation are the optimum concentration to decontaminate sputum so both conditions not only can prevent the growth of contaminant but also support recovery of TB bacilli.

\section{CONCLUSION}

Bleach at $1 \%$ concentration have a optimal positivity rate as good as common decontaminant such as $4 \% \mathrm{NaOH}$ and $2 \%$ NALC-NaOH especially for AFB score higher than (+2).

\section{ACKNOWLEDGMENT}

Thank you to the all staffs of TB laboratory of Microbiology Laboratory of Medical Faculty University of Indonesia. This research was funded by funding grant from Center of Publication and Research of Syarif Hidayatullah State Islamic University (UIN) Jakarta.

\section{REFERENCES}

[1] WHO. WHO, 2016: Global Tuberculosis Raport. 2016.

[2] Pyffer GE, Palicova F. Mycobacterium: General Characteristics, Laboratory Detection and Staining Procedurs. In: Manual of Clinical Microbiology. ; 2011:472-524.

[3] Warner DF, Koch A, Mizrahi V. Diversity and disease pathogenesis in Mycobacterium tuberculosis. Trends Microbiol. 2015;23(1):14-21. doi:10.1016/j.tim.2014.10.005.

[4] Chatterjee M, Bhattacharya S, Karak K, Dastidar SG. Effects of different methods of decontamination for successful cultivation of Mycobacterium tuberculosis. Indian J Med Res. 2013;138(OCT). doi:IJMR-138-541 [pii].

[5] Yajko DM, Nassos PS, Sanders CA, et al. Comparison of four decontamination methods for recovery of Mycobacterium avium complex from stools. J Clin Microbiol. 1993;31(2):302-306.

[6] Peres RL, Maciel ELN, Morais CG, et al. Comparison of two concentrations of NALC-NaOH for decontamination of sputum for mycobacterial culture. Int J Tuberc Lung Dis. 2009;13(12):15721575.

[7] Satapathy P, Das D, Murmu BN, Kar SK. Decontamination of sputum for longer time in sodium hydroxide for isolation of Mycobacterium tuberculosis. Int $J$ Mycobacteriology. 2014;3(4):290-292. doi:10.1016/j.ijmyco.2014.09.006.

[8] Sula L. Comparative trials with different decontaminating agents for growing Mycobacterium tuberculosis from sputum specimens. Bull World Health Organ. 1968;39(5):647-655.

[9] McClean M, Stanley T, Stanley S, et al. Identification and characterization of breakthrough contaminants associated with the conventional isolation of Mycobacterium tuberculosis. J Med Microbiol. 2011;60(9):1292-1298. doi:10.1099/jmm.0.030619-0.

[10] Sharma M, Misra R, Gandham N, Jadhav S, Angadi K, Wilson V. Comparison of modified Petroff's a nd N-acetyl-Lcysteine-sodium hydroxide methods for sputum decontamination in tertiary care hospital in India. Med $J$ Dr DY Patil Univ. 2016;5(2). doi:10.4103/0975-2870.103323.

[11] Cheon SA, Cho HH, Kim J, Lee J, Kim HJ, Park TJ. Recent tuberculosis diagnosis toward the end TB strategy. J Microbiol Methods. 2016;123:51-61. doi:10.1016/j.mimet.2016.02.007.

[12] Winter J, Ilbert M, Graf PCF, Özcelik D, Jakob U. Bleach Activates a Redox-Regulated Chaperone by Oxidative Protein Unfolding. Cell. 2008;135(4):691-701. doi:10.1016/j.cell.2008.09.024

[13] Kassaza K, Orikiriza P, Llosa A, et al. Lowenstein-Jensen selective medium for reducing contamination in Mycobacterium tuberculosis culture. J Clin Microbiol. 2014;52(7). doi:10.1128/JCM.00749-14.

[14] Karakeçe E, Terzi HA, Çiftci IH. Could a step in the isolation of mycobacteria from sputum samples be eliminated by new decontamination kits? Polish J Microbiol. 2014;63(3):369-371.

[15] Fitzgerald Daniel W, Starling Timothy R HDW. Mycobacterium tuberculosis. Eighth Edi. Elsevier Inc.; 2010. doi:10.1016/B978-1- 
4557-4801-3.00251-4. 